Abstract 218 Table 1 Multivariate analysis for overall survival. Recurrence-free survival, and disease-free survival of cervical cancer patients
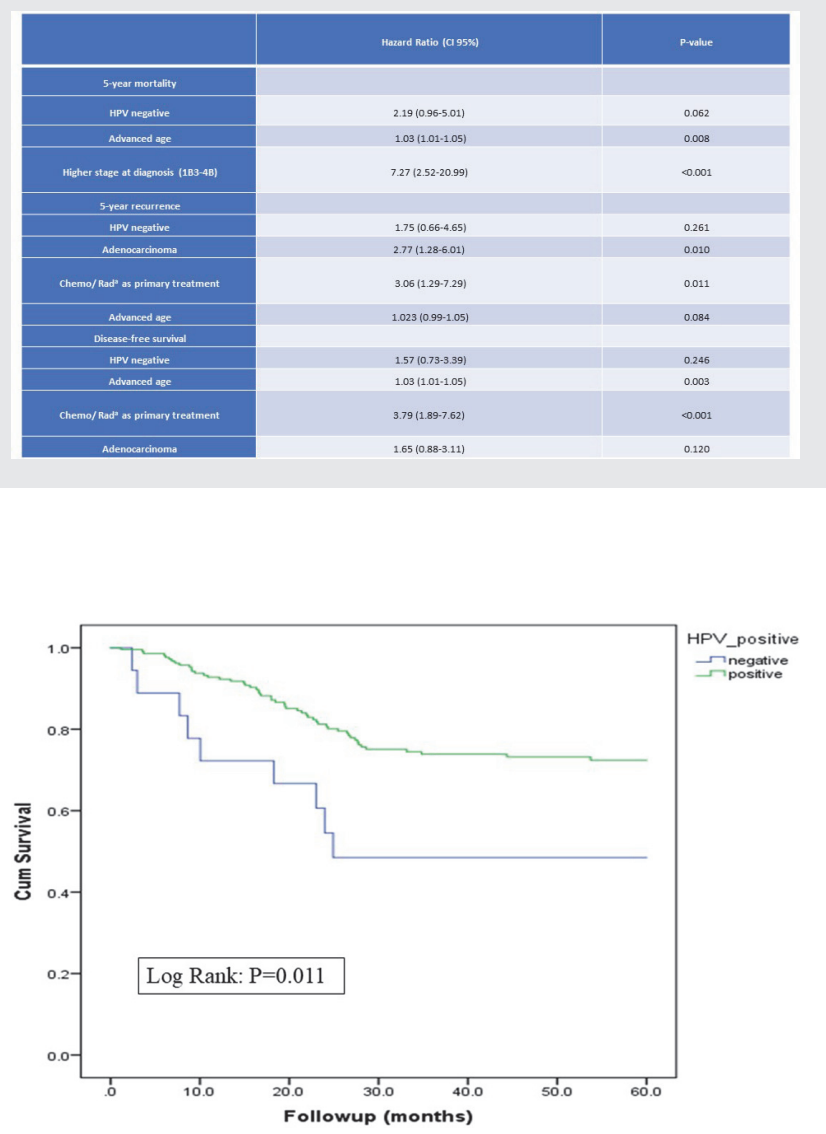

Abstract 218 Figure 1 Kaplan Meier curve of overall survival, comparing women with HPV-negative and HPV-positive cervival cancer

negativity $(P=0.062)$. Median overall survival for HPV-positive CC was not reached, compared to 24 months for HPV-negative CC. Kaplan-Meier curves showed lower rates of overall survival $(P=0.011)$, recurrence-free survival $(P=0.005)$ and disease-free survival $(P=0.008)$ for women with HPV-negative compared to HPV-positive CC.

Conclusion* The relatively poor prognosis of HPV-negative CC is important in light of its relatively high prevalence, which could increase proportionally to HPV-positive CC due to increased HPV screening and vaccination. Further studies are needed to confirm if HPV status is truly an independent prognostic factor in CC.

\section{CLEARANCE OF HPV AFTER CONIZATION OF CERVICAL CANCER AND ADENOCARCINOMA IN SITU CORRELATES WITH ABSENCE OF CANCER}

${ }^{1} \mathrm{~N}$ Kugelman*, ${ }^{1} \mathrm{E}$ Siegler, ${ }^{1} Y$ Goldberg, ${ }^{2} Y$ Siegler, ${ }^{3} \mathrm{P}$ Shaked Mishan, ${ }^{4} \mathrm{~S}$ Mazareb, ${ }^{1} \mathrm{~L}$ Mackuli, ${ }^{4} \mathrm{E}$ Sabo, ${ }^{2} \mathrm{O}$ Lavie, ${ }^{2} Y$ Segev. ${ }^{1}$ Carmel Medical Center, Obstetrics and Gynecology, Rappaport Faculty of Medicine, Technion-Israel Institute of Technology, Haifa, Israel; ${ }^{2}$ Rambam Health Care Campus, Obstetrics and Gynecology, Rappaport Faculty of Medicine, Technion-Israel Institute of Technology, Haifa, Israel; ${ }^{3}$ Carmel Medical Center, Clinical Serology and Virology Laboratory, Haifa, Israel; ${ }^{4}$ Carmel Medical Center, Pathology, Haifa, Israel

10.1136/ijgc-2021-ESG0.19
Introduction/Background* About $40 \%$ of CC are in women under age 40 years, for whom fertility-sparing treatment might be very important and should always be considered. More than half the patients who undergo radical surgery (trachelectomy or hysterectomy) have no remnant tumor in the final pathology specimen. HPV clearance is a test of cure after conisation, but it is not a test of cure in CC. Our objective was to assess correlation of clearance of high-risk human papillomavirus (HR-HPV) after large loop excision of the transformation zone (LLETZ) with absence of residual disease, in women diagnosed with cervical cancer (CC) and Adenocarcinoma in Situ (AIS).

Methodology Data was collected from 92 women diagnosed with CC and AIS who were positive to High -Risk HPV (HR-HPV), and had a repeat cervical HPV test 3-12 weeks post-LLETZ, and before final surgical treatment. We compared characteristics of women with negative and positive HR-HPV post-LLETZ.

Result(s)* The pathological results of women who were HRHPV negative $(n=40)$ compared to HR-HPV positive $(n=52)$ at the post-LLETZ follow-up visit included a significantly higher incidence of AIS: $14(35 \%)$ vs 5(9.6\%) $(\mathrm{p}<0.006)$. In the negative HR-HPV post-LLETZ group, 36 (90\%) had normal histology and only $2(5 \%)$ had cancer in the final histological specimen. Among women who underwent radical hysterectomy/trachelectomy after LLETZ, a normal final histology was observed in $75 \%$ and $9 \%$ of those who were HRHPV negative and HR-HPV positive, respectively $(\mathrm{p}<0.0005)$. The negative predictive value for residual cancer, with clearance of HR-HPV after LLETZ was 95\%.

Conclusion* Clearance of HR-HPV from the cervix a short time after LLETZ has a high correlation with the absence of residual cancer in the final outcome, either in the pathology or the follow up. Testing for HR-HPV a short time after LLETZ might serve as a parameter for risk assessment.

\section{THE SIGNIFICANCE OF SURGICAL ASSESSMENT IN ONCOLOGICAL OUTCOMES AFTER RADICAL HYSTERECTOMY FOR EARLY-STAGE CERVICAL CANCER. A MULTICENTER STUDY}

${ }^{1} S$ Fernandez-Gonzalez*, ${ }^{2} \mathrm{M}$ Barahona, ${ }^{3} \mathrm{~A}$ Gil-Moreno, ${ }^{3} \mathrm{NR}$ Gómez-Hidalgo, ${ }^{4} \mathrm{~B}$ DiazFeijoo, ${ }^{5} \mathrm{P}$ Coronado, ${ }^{5} \mathrm{~V}$ González, ${ }^{5} \mathrm{~A}$ Casajuana, ${ }^{6} \mathrm{JM}$ Silvan, ${ }^{6} \mathrm{~L}$ Melero, ${ }^{6} \mathrm{MA}$ Martinez, ${ }^{7} \mathrm{~J}$ Hilario de la Rosa, ${ }^{7} \mathrm{I}$ Lobo, ${ }^{7} \mathrm{E}$ Beiro, ${ }^{8} \mathrm{~J}$ Frias-Gomez, ${ }^{8} \mathrm{Y}$ Benavente, ${ }^{2} \mathrm{C}$ Ortega, ${ }^{9} \mathrm{E}$ Martinez, ${ }^{2} \mathrm{~L}$ Marti, ${ }^{2} \mathrm{~J}$ Ponce. ${ }^{1}$ University Hospital of Bellvitge (IDIBELL), Gynecology, L'Hospitalet de Llobregat, Spain; '2University Hospital of Bellvitge (IDIBELL), Gynecology, L'Hospitalet de Llobregat (Barcelona), Spain; ${ }^{3}$ Hospital Universitari Vall d'Hebron, Department of Gynecologic Oncology, Barcelona; ${ }^{4}$ Hospital Clínic de Barcelona, Gynecology, Barcelona, Spain; ${ }^{5}$ Hospital Clínico San Carlos, Instituto de Salud de la Mujer, Madrid, Spain; ${ }^{6}$ Hospital Universitario Virgen del Rocio, Gynecology, Sevilla, Spain; ${ }^{7}$ Hospital Universitario de Basurto, Gynecology, Bilbao, Spain; ${ }^{8}$ Catalan Institute of Oncology (IDIBELL), Cancer Epidemiology Research Programme, L'Hospitalet de Llobregat, Spain ${ }^{9}$ University Hospital of Bellvitge (IDIBELL), Nursery, L'Hospitalet de Llobregat (Barcelona), Spain, Spain

\subsection{6/ijgc-2021-ESGO.20}

Introduction/Background* Patients with early-stage cervical cancer [CC] had worse prognosis when operated by minimal invasive surgery according to LACC trial. Different hypothesis were suggested such us the intrauterine manipulator, the $\mathrm{CO} 2$ or the lack of protective manoeuvre. However, the effect of surgical expertise among patients who underwent radical hysterectomy by the same approach has not been evaluated. 
Methodology All patients with early-stage CC (FIGO IA1-IIA1) undergoing robot-assisted radical hysterectomy in Spain and Portugal from 2009 to 2018 were included. Those centres with $>15$ cases were selected. Centres with recurrence rate $<10 \%$ were gathered in group $\mathrm{A}$ an those $\geq 10 \%$ in group B. The primary objective was to compare the oncological outcomes between groups after balancing by Propensity Score [PS] analysis. The groups were balanced in age, BMI, histology, Size, tumoral grade, ILV and adjuvant treatment. Second primary objective was to audit the pre-surgical quality indicators [QI] proposed by ESGO.

Result(s)* A total of 118 and 97 patients were well balanced (p-value 0.9483) between groups. 5 (4.3\%) vs 19 (19.6\%) recurrences occurred in group A vs group B, OR 1.23; $(95 \%$ CI, 1,13-1,35) p-value of 0.001 after a median follow-up of 51 months. Overall mortality and disease-specific mortality were significant higher in group B, OR 1.07; (95\% CI, 1.001.15 ) and 1.09; (95\% CI, 1.02-1.16) respectively (figure 1). Five of eight Q.I were fulfilled by both groups. Lower rates of pre-operative assessment with M.R.I was observed in group B. $1(20 \%)$ and $8(42 \%)$ recurrences were observed during the first two years of robotic experience in group A and B (figure 2). Intraoperative and postoperative complications occurred in 0.8 vs $6.2 \%$ (p 0.028 ) and $5.1 \%$ vs $12.4 \%$ (p $0.055)$ in groups $A$ and $B$ respectively.

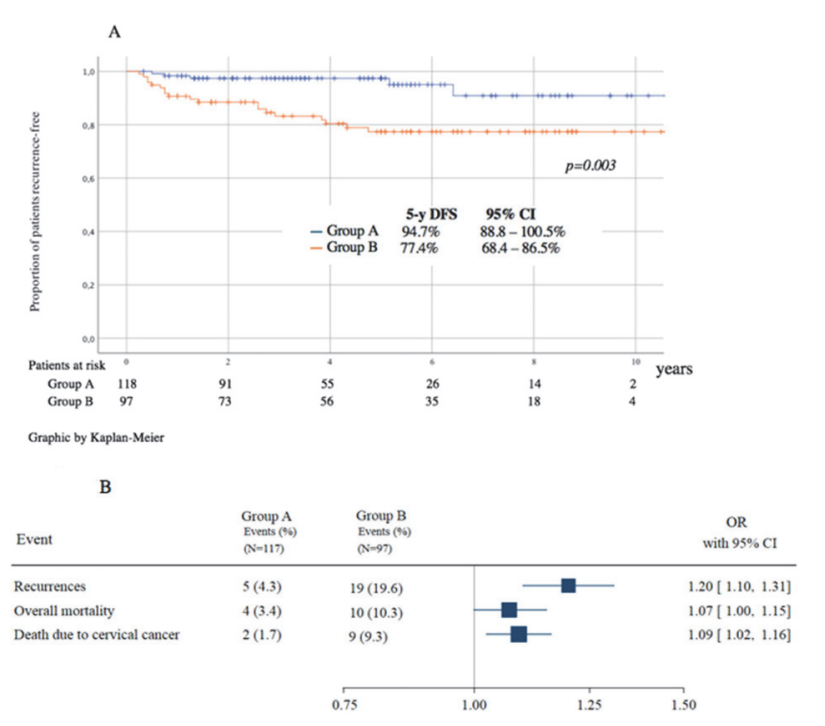

Abstract 245 Figure 1 A) Disease-free survival rates between surgical; B) Odds of recurrence \& mortality after balancing
Conclusion* We observed significant differences in recurrence rate, overall mortality and specific-disease mortality between hospitals. Pre-operative assessment with M.R.I and the effect of learning curve were factors related to higher rates of recurrence. The surgical assessment might be considered as an impact factor in oncological outcomes in patients who underwent radical hysterectomy by minimal invasive approach.

\section{ENGOT-CX11/GOG 3047/KEYNOTE-A18: PHASE 3 RANDOMIZED STUDY OF PEMBROLIZUMAB + CHEMORADIOTHERAPY FOR HIGH-RISK LOCALLY ADVANCED CERVICAL CANCER}

${ }^{1} \mathrm{D}$ Lorusso* ${ }^{2} Y$ Xiang, ${ }^{3} \mathrm{~N}$ Colombo, ${ }^{4} \mathrm{RL}$ Coleman, ${ }^{5} \mathrm{LM}$ Randall, ${ }^{6} \mathrm{~L}$ Duska, ${ }^{7} \mathrm{~K}$ Hasegawa, ${ }^{8} \mathrm{~A}$ Nogueira-Rodrigues, ${ }^{9} \mathrm{D}$ Cibula, ${ }^{10} \mathrm{MR}$ Mirza, ${ }^{11} \mathrm{~B}$ You, ${ }^{12} \mathrm{~A}$ Oaknin, ${ }^{13} \mathrm{M}$ Christiaens, ${ }^{14} \mathrm{C}$ Taskiran, ${ }^{15} \mathrm{~J}$ Sehouli, ${ }^{16} \mathrm{~J}$ Korach, ${ }^{17} \mathrm{C}$ Marth, ${ }^{18} \mathrm{~K}$ Yamada, ${ }^{18} \mathrm{M}$ Puglisi, ${ }^{19} \mathrm{~S}$ Pignata. ${ }^{1}$ Associate Professor of Obstetrics and Gynecology, Catholic University of Sacred Heart; Clinical Research Development Unit Fondazione Policlinico Gemelli IRCCS, Rome, Italy; ${ }^{2}$ Department of Gynecology and Obstetrics, Peking Union Medical College Hospital, Chinese Academy of Medical Sciences, Beijing, China; ${ }^{3}$ Department of Gynecology, Instituto Europeo di Oncologia, Milan, Italy; ${ }^{4}$ Department of Gynecologic Oncology and Reproductive Medicine, University of Texas MD Anderson Cancer Center, Houston, TX, USA; ${ }^{5}$ Department of Obstetrics and Gynecology, Massey Cancer Center, Virginia Commonwealth University, Richmond, VA, USA; ${ }^{6}$ Department of Obstetrics and Gynecology, University of Virginia, Charlottesville, VA, USA; ${ }^{7}$ Department of Gynecologic Oncology, Saitama Medical University, Hidaka, Saitama Prefecture, Japan; ${ }^{8}$ Departamento de Clínica Médica, Universidade Federal de Minas Gerais, Belo Horizonte, Minas Gerais, Brazil; ${ }^{9}$ Department of Obstetrics and Gynecology, General Faculty Hospital in Prague, First Faculty of Medicine, Charles University, Prague, Czech Republic; ${ }^{10}$ Department of Oncology, Copenhagen University Hospital, Copenhagen, Denmark; ${ }^{11}$ Department of Medical Oncology, CITOHL, IC-HCL, Hospices Civils de Lyon, Université Claude Bernard Lyon 1, Lyon, France; ${ }^{12}$ Department of Medical Oncology, Vall d'Hebron Institute of Oncology (VHIO), Hospital Universitari Vall d'Hebron, Vall d'Hebron Barcelona Hospital Campus, Barcelona, Spain; ${ }^{13}$ Department of Radiation Oncology, Universitair Ziekenhuis Leuven, Leuven, Belgium; ${ }^{14}$ Professor Koc University School of Medicine and VKV American Hospital, Department of Obstetrics and Gynecology, Division of Gynecologic Oncology and Turkish Society of Gynecologic Oncology, Istanbul, Turkey; ${ }^{15}$ Deparment of Gynecology, Charité-Universitätsmedizin Berlin, Berlin, Germany; ${ }^{16}$ Gynecology Oncology Department, Sheba Medical Center, Ramat Gan, Israel; ${ }^{17}$ Department of Obstetrics and Gynecology, Medizinische Universität Innsbruck, Innsbruck, Austria; ${ }^{18}$ Clinical Development, Merck and Co., Inc., Kenilworth, NJ, USA; ${ }^{19}$ Department of Uro-Gynaecological Oncology, Instituto Nazionale Tumori IRCCS Fondazione G Pascale, Italy

\subsection{6/ijgc-2021-ESG0.21}

Introduction/Background* High-risk locally advanced cervical cancer has a poor prognosis, and more than half of patients recur in 2 years. External beam radiotherapy (EBRT) with concurrent chemotherapy followed by brachytherapy is the standard of care for locally advanced cervical cancer. The

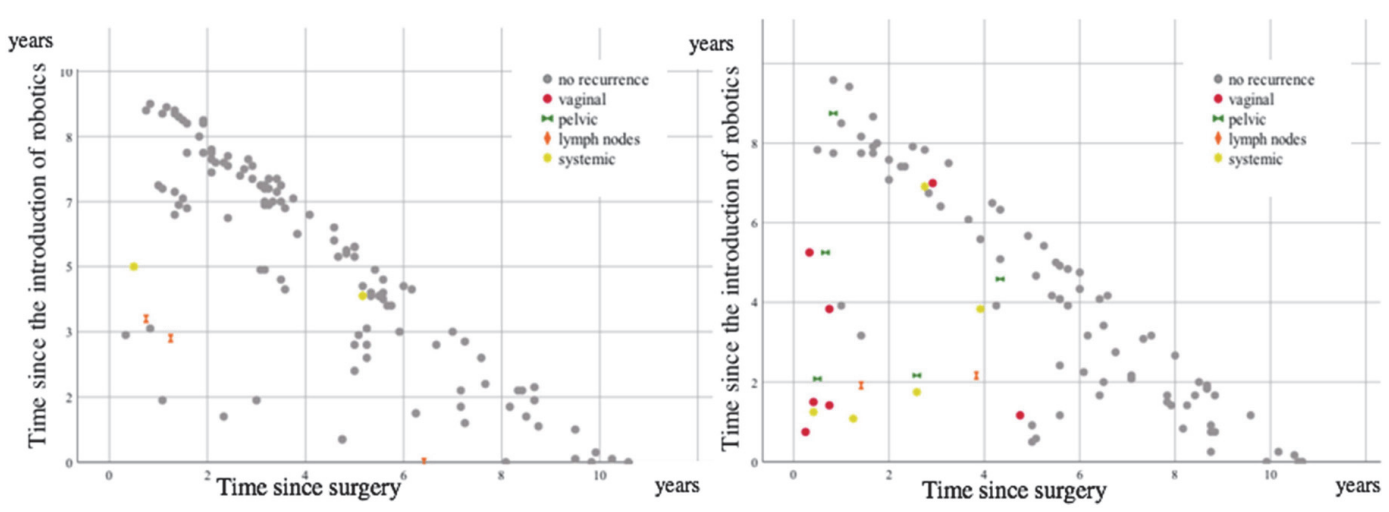

Abstract 245 Figure 2 (Group A and B) relation between time of recurrence and time of surgery 